\title{
ON THE MAXIMAL DILATION OF QUASICONFORMAL MAPPINGS
}

KURT STREBEL

1. Let $G$ and $G^{\prime}$ be two plane open sets and $w(z)$ a topological mapping of $G$ onto $G^{\prime}$. By $Q$ we denote any quadrilateral in $G$, i.e. the topological image of a closed square with a distinguished pair of opposite sides. The conformal modulus $m$ of $Q$ is the ratio $m=a / b$ of the sides of a conformally equivalent rectangle $R$, the distinguished sides of $Q$ corresponding to the sides of length $b$. We call this essentially unique conformal mapping of $Q$ onto $R$ the canonical mapping of $Q$. The modulus $m$ is equal to the extremal distance of the two distinguished sides of $Q$ with respect to $Q$. The maximal dilation of the mapping $w(z)$ on $G$ is the number

$$
K[w(z)]=K=\sup _{\mathbf{Q}} \frac{m^{\prime}}{m}
$$

where $m^{\prime}$ denotes the modulus of the image $Q^{\prime}$ of $Q$ under the mapping $w(z)$ and $Q$ varies over all possible quadrilaterals. The mapping is said to be quasiconformal if $K$ is finite.

Given a closed subset $E \subset G$ (closed only with respect to $G$ ). Then, $G-E$ is open, and if we denote by $K_{0}[w(z)]=K_{0}$ the maximal dilation of $w(z)$ on $G-E$, we get $K_{0} \leqq K$. We are looking for sufficient conditions on $E$ such that $K_{0}=K$. The answer will be different if we consider only the class of all quasi-conformal mappings of $G$ or the larger class of all topological mappings, including the ones with infinite maximal dilation. We call a point set $E$ which allows the conclusion $K_{0}=K$ deletable for the class in consideration. It was proved by Ahlfors in [1] that analytic arcs are deletable for the class of all topological mappings. ${ }^{1}$ It is also proved there that $K=\sup \widetilde{Q} m^{\prime} / m$, where $\tilde{Q}$ denotes any analytic quadrilateral, i.e. a quadrilateral $Q$ with a canonical mapping which is conformal in an open neighborhood of $Q$, and furthermore that $K$ does not become larger if the boundary curves of the quadrilaterals are allowed to have points in common with the boundary of $G$.

2. If $E$ is a discrete point set, we can consider the slightly more general problem that $w_{0}(z)$ is only known to be quasiconformal with maximal dilation $K_{0}$ on $G-E$, without knowing that $w_{0}(z)$ is a topological mapping of the whole open set $G$. What are the conditions on

Presented to the Society, February 26, 1955; received by the editors November 8 , 1954 and, in revised form, December 31, 1954.

1 Theorem 4, p. 9. 
$E$ in order that for every $w_{0}(z)$ there is a topological mapping $w(z)$ of $G$ which coincides with $w_{0}(z)$ on $G-E$ and has the same maximal dilation. We call $w(z)$ the continuation of $w_{0}(z)$. It is clear that there can exist only one topological continuation.

THEOREM 1. A necessary and sufficient condition that every quasiconformal mapping $w_{0}(z)$ of $G-E$ has a continuation to $G$ with the same maximal dilation is that every compact subset of $E$ is a nullset $O_{A D}{ }^{2}$

The condition is necessary, for, if $E_{0}$ is any compact subset of $E$ which is not a set $O_{A D}$, there exists a parallel slit mapping of the complement of $E_{0}$, which is conformal outside $E_{0}$ and cannot be extended conformally over $E_{0}$.

If, on the other hand, $E$ possesses the property of the theorem, then to every point $z_{0} \subset E$ there exists a sequence of nonoverlapping doubly connected ring-domains in $G-E$ with a divergent sum of moduli $\mu_{i}\left(\mu_{i}=\right.$ extremal distance of the two boundary components of a ring domain), a property which is invariant under the quasiconformal mapping of $G-E$. But a boundary component with this property must necessarily be a point. Therefore every point of $E$ goes over into a point, and it is readily seen that $w_{0}(z)$ has a topological continuation $w(z)$ over $E$. It follows from the succeeding lemma that the maximal dilation of $w(z)$ on $G$ is $K_{0}$.

Lemma 1. If $Q$ is an analytic quadrilateral in $G$ and $E_{0}$ a compact $O_{A D}$ set in $Q$, then to every $\epsilon>0$ there exists a finite system of simply connected, nonoverlapping longitudinal ${ }^{3}$ strips $S_{i}, S_{i} \subset Q-E_{0}$ with modulus $m_{i}$, such that $\sum_{i} 1 / m_{i} \geqq 1 / m-\epsilon$.

From that we get, because of $m_{i}^{\prime} \leqq K_{0} m_{i}$,

$$
\frac{1}{m^{\prime}} \geqq \sum_{i} \frac{1}{m_{i}^{\prime}} \geqq K_{0}^{-1} \sum_{i} \frac{1}{m_{i}} \geqq K_{0}^{-1}\left(\frac{1}{m}-\epsilon\right)
$$

and therefore

$$
m^{\prime} \leqq K_{0} m
$$

for each analytic quadrilateral in $G$.

The lemma can be proved in the same way as Theorem 9 in Ahlfors and Beurling [2]. We map $Q$ onto a rectangle $R$ with sides $a$ and $b$ by means of its canonical conformal mapping. $E_{0}$ is transformed

${ }^{2}$ I.e. a set which allows no nonconstant and single-valued analytic function with a bounded Dirichlet-integral in its complement.

3 That is to say the boundary of $S_{i}$ has an interval in common with each distinguished side of $Q ; S_{i}$ is therefore a quadrilateral with these intervals as distinguished sides. 
into a set $O_{A D}$ which we denote by $E_{0}^{r}$. To any given $\epsilon>0$ we can find a concentric rectangle $R^{\prime}$ with sides $a^{\prime}>a$ and $b^{\prime}<b$ and such that $b^{\prime} / a^{\prime} \geqq b / a-\epsilon / 2$. A curvilinear rectangle $R^{\prime \prime}$ which is contained in the rectangle with sides $a^{\prime}, b$ and contains the rectangle with sides $a, b^{\prime}$ and the sides of which do not meet $E_{0}^{\prime}$ can be constructed; we choose its distinguished sides outside $R$ and such that its modulus is $\leqq a^{\prime} / b^{\prime}$. The set $E_{0}^{\prime} \cap R^{\prime \prime}$ is a compact subset of the open rectangle $R^{\prime \prime}$, and by an exhaustion we can obviously find the strips $S_{i}^{\prime \prime}$ in $R^{\prime \prime}$ with $\sum_{i} 1 / m_{i}^{\prime \prime} \geqq 1 / m^{\prime \prime}-\epsilon / 2$. Each $S_{i}^{\prime \prime}$ contains a longitudinal strip $S_{i}$ of the original rectangle $R$ with modulus $m_{i} \leqq m_{i}^{\prime \prime}$. Therefore we get

$$
\sum_{i} \frac{1}{m_{i}} \geqq \sum_{i} \frac{1}{m_{i}^{\prime \prime}} \geqq \frac{1}{m^{\prime \prime}}-\epsilon / 2 \geqq b^{\prime} / a^{\prime}-\epsilon / 2 \geqq b / a-\epsilon=\frac{1}{m}-\epsilon .
$$

3. For the composition of piecewise quasiconformal mappings however the stress lies on connected, not on discrete point sets. To get an answer in this direction, we consider a rectangle $R$ in the $z$-plane $(0 \leqq x \leqq a, 0 \leqq y \leqq b)$ and a topological mapping $w(z)$ of $R$ onto a rectangle $R^{\prime}$ in the $w$-plane $\left(0 \leqq u \leqq a^{\prime}, 0 \leqq v \leqq b^{\prime}\right)$ which preserves the four sides respectively. By $E_{y}$ we denote the intersection of the line $\Im_{z}=y$ with the given closed set $E$ in $R$, and by $L_{u}(y)$ the linear measure of the vertical projection (i.e. onto the $u$-axis) of the $w$-image $E_{y}^{\prime}$ of $E_{y}$. The modulus of a horizontal rectangle is its length divided by its height; the modulus of its w-image has to be taken with respect to the sides which correspond to the vertical sides of the rectangle.

LEMMA 2. If (1) the modulus $m$ of every horizontal rectangle in $R$ which has no interior point in common with $E$ and the modulus $m^{\prime}$ of its image satisfy $m^{\prime} \leqq K m$, where $K$ is some positive constant, and

(2) the linear measure $L_{u}(y)$ of the vertical projection of $E_{\nu}^{\prime}$ is zero for almost every $y$, we have

$$
a^{\prime} / b^{\prime} \leqq K a / b \text {. }
$$

Proof. For any $\epsilon>0$ the set $O_{\epsilon}$ of all values $y$ with $L_{u}(y)<\epsilon$ is open in $0 \leqq y \leqq b$ and has linear measure $b$. For any $y \in O_{\epsilon}$ we can find an interval $y_{1}<y<y_{2}$ and a family of finitely many rectangles $R_{i}$ with sides on $\Im_{z}=y_{1}$ and $\Im_{z}=y_{2}$ which contain every $E_{y}$ for $y_{1}<y<y_{2}$ and such that their w-images have a vertical projection of total linear measure less than $\epsilon$. Let now $B$ be a closed subset of $O_{\epsilon}$ of measure $>b-\epsilon$. We have an open covering of $B$ by means of intervals $\beta$ of the above kind, and by the Heine-Borel theorem there exists a finite covering, say $\beta_{1}, \cdots, \beta_{n}$. Starting with $\beta_{1}$ and the rectangles in the corresponding horizontal strip $\left(0 \leqq x \leqq a, y \in \beta_{1}\right)$, we take in 
every new interval $\beta_{i}$ only that part which does not lie in one of the former intervals, and restrict the corresponding horizontal strip and its rectangles in the same way. We get a system of finitely many strips $S_{i}$ of height $b_{i}$ with total vertical measure $\sum_{i} b_{i} \geqq b-\epsilon$. The rectangles $T_{i j}$ in $S_{i}$, complementary to the rectangles $R_{i j}$ in $S_{i}$, have no interior point in common with $E$ and thus their moduli satisfy $m_{i j}^{\prime} \leqq K m_{i j}$. As the vertical projection of the images $R_{i j}^{\prime}$ of the $R_{i j}$ has a total linear measure $<\epsilon$, there exist finitely many closed, disjoint intervals on the $u$-axis of total measure $<\epsilon$ covering this projection. We denote the complementary intervals on the $u$-axis by $\alpha_{i j}^{\prime}$, their length by $a_{\hat{j}}^{\prime}$ : we have $\sum_{j} a_{i j}^{\prime}>a^{\prime}-\epsilon$. Each $\alpha_{i j}^{\prime}$ is spanned by the image $T_{i j}^{\prime}$ of at least one $T_{i j}$, and the modulus of this $T_{i j}^{\prime}$ is therefore $\geqq a_{i j}^{\prime 2} / A_{i j}^{\prime}, A_{i j}^{\prime}$ denoting the area of (the interior of) $T_{i j}^{\prime}$. From that we get for each strip $S_{i}$

$$
\begin{aligned}
\frac{a}{b_{i}} & \geqq \sum_{j} m_{i j} \geqq \frac{1}{K} \sum_{j} m_{i j}^{\prime} \geqq \frac{1}{K} \sum_{j} \frac{a_{i j}^{\prime 2}}{A_{i j}^{\prime}} \\
& \geqq \frac{1}{K}\left(\sum_{j} a_{i j}^{\prime}\right)^{2} / \sum_{j} A_{i j}^{\prime} \geqq \frac{1}{K} \frac{\left(a^{\prime}-\epsilon\right)^{2}}{A_{i}^{\prime}}
\end{aligned}
$$

with $A_{i}^{\prime}=$ area of $S_{i}^{\prime}$. Taking the reciprocals and summing up we get

$$
\frac{b-\epsilon}{a} \leqq \frac{\sum_{i} b_{i}}{a} \leqq K \frac{\sum_{i} A_{i}^{\prime}}{\left(a^{\prime}-\epsilon\right)^{2}} \leqq K \frac{a^{\prime} b^{\prime}}{\left(a^{\prime}-\epsilon\right)^{2}}
$$

and therefore

$$
b / a \leqq K\left(b^{\prime} / a^{\prime}\right)
$$

which proves the lemma.

Let $E$ be an arbitrary set of finite linear measure $L$ in $R$. Then it is readily proved that the set of values $y$ where $E_{y}$ consists of at least $N$ points has linear measure $\leqq L / N$, and therefore the set of $y$ where $E_{y}$ consists of infinitely many points has measure zero. If $E$ is of $\Sigma$-finite measure, i.e. the sum of denumerably many sets $E^{i}$ of finite linear measure, the set of values $y$ for which $E_{y}$ consists of nondenumerably many points has linear measure zero. But for any $y$ where $E_{y}$ is denumerable, the image set is denumerable and so is its projection, therefore $L_{u}(y)=0$. As the property to be of $\Sigma$-finite linear measure is carried over by a conformal mapping of the closed quadrilateral, we get the

THEOREM 2. If $E$ is a closed subset of $G$ of $\Sigma$-finite linear measure and $w(z)$ any topological mapping of $G$, its maximal dilation on $G$ is equal 
to its maximal dilation on $G-E$, that is to say $E$ is deletable for the class of all topological mappings of $G$.

If $E=E^{1}+E^{2}$ is the sum of a closed set $E^{1}$ of $\Sigma$-finite linear measure and a closed set $E^{2}$, every compact subset of which is a nullset $O_{A D}$, then it is clear from the construction that $E$ is deletable. We can first delete $E^{1}-E^{2}$, which is a closed set of $G-E^{2}$, and then $E^{2}$.

From Theorem 2 we get the following generalization of Theorem 13 in Ahlfors and Beurling [2]:

Corollary. A closed, discrete point set $E$ of the complex plane, which is of $\Sigma$-finite linear measure, is a nullset $O_{S_{B}}{ }^{4}$ if and only if it is a nullset $O_{A D}$.

For, if it is a nullset $O_{S B}$ every schlicht conformal mapping of the complement of $E$ is continuous on $E$ and has therefore a conformal continuation, i.e. is a linear transformation. But this is known to be a sufficient condition for a closed discrete pointset to be an $O_{A D}$ set. The converse is obvious.

4. If the mapping $w(z)$ is not only known to be topological in $G$ but quasiconformal, that means has finite maximal dilation, and if outside $E$ the maximal dilation is $K_{0}$, the point sets $E$ which allow us to conclude $K_{0}=K$ are much larger.

Lemma 3. Let w(z) be a topological mapping of $R$ onto $R^{\prime}$ as in Lemma 2. If the two following conditions are fulfilled:

(1) For every horizontal rectangle in $R$ and a certain positive number $K$ we have $m^{\prime} \leqq K m$;

(2) $E$ is of two-dimensional measure zero; then the linear measure $L(y)$ of $E_{y}^{\prime}$ is zero for almost every $y$.

Proof. If this were not the case, we could find a closed subset $B$ of $0 \leqq y \leqq b$ of positive linear measure $h$ and a positive number $l$ such that the linear measure of $E_{y}$ would be zero while the linear measure of $E_{y}^{\prime}$ would be $L(y) \geqq l$ for every $y \in B$. This is so because $L(y)$ is a measurable function of $y$ and the linear measure of $E_{y}$ is zero for almost all $y$.

If $y \in B$ is arbitrary, we can cover $E_{y}$ by finitely many open (relatively to $0 \leqq x \leqq a)$ intervals $\alpha_{j}$ of length $a_{j}$. The images have length $a_{j}^{\prime}$. We then take an interval $y_{1}<y<y_{2}$ and consider the rectangles $R_{j}\left(x \in \alpha_{j}, y_{1} \leqq y \leqq y_{2}\right)$. We call them the rectangles corresponding to the intervals $\alpha_{j}$ in the horizontal strip $\left(0 \leqq x \leqq a, y_{1} \leqq y \leqq y_{2}\right)$. Every $R_{j}$ is mapped onto a quadrilateral $R_{j}^{\prime}$ and we denote by $l_{j}$ the inf.

- I.e. the complement allows no schlicht, bounded conformal mapping. 
of the length of all curves joining the two distinguished sides in $R_{j}^{\prime}$, by $F_{j}$ the area of the open $R_{j}^{\prime}$. Because $E_{y}$ is of measure zero and $\sum_{j} a_{j}^{\prime} \geqq l$, it is clear that, given any $y \in B$, we can choose the $\alpha_{j}$ and afterwards $y_{1}<y<y_{2}$ in such a way that

$$
\sum_{j} a_{j} \leqq \epsilon \text { and } \sum l_{j} \geqq l-\epsilon .
$$

The intervals $y_{1}<y<y_{2}$ provide us with an open covering of $B$ from which we get a finite covering. As in Lemma 2 the restriction to distinct strips $S_{i}$ does not change the above two conditions, and we have for any strip $S_{i}$ (with its intervals $\alpha_{i j}$ of length $a_{i j}$ and the corresponding rectangles $R_{i j}$ with moduli $m_{i j}$ ) the following estimates:

$$
\frac{a_{i j}}{b_{i}}=m_{i j} \geqq \frac{1}{K} m_{i j}^{\prime} \geqq \frac{1}{K} \frac{l_{i j}^{2}}{F_{i j}} .
$$

Therefore

$$
\frac{\epsilon}{b_{i}} \geqq \sum_{j} a_{i j} / b_{i} \geqq \frac{1}{K} \sum_{j} \frac{l_{i j}^{2}}{F_{i j}} \geqq \frac{1}{K}\left(\sum_{j} l_{i j}\right)^{2} / \sum_{j} F_{i j} \geqq \frac{1}{K} \frac{(l-\epsilon)^{2}}{\sum_{j} F_{i j}} .
$$

Taking reciprocals and summing up we get

$$
\frac{h}{\epsilon} \leqq \sum_{i} b_{i} / \epsilon \leqq K \sum_{i j} F_{i j} /(l-\epsilon)^{2} \leqq K \frac{a^{\prime} b^{\prime}}{(l-\epsilon)^{2}} .
$$

As $\epsilon \rightarrow 0$ we conclude $h=0$, q.e.d.

With exactly the same method but less rough estimates we can get the following result:

Lemma $3^{\prime}$. Let $E$ be any closed set in $R, B$ a closed subset of $0 \leqq y \leqq b$ of measure $h$ and such that for $y \in B$ the linear measure of $E_{y}$ is $\leqq l$ while the linear measure of $E_{y}^{\prime}$ is $L(y) \geqq l^{\prime}$. Let $F$ denote the area of the closed set $\bigcup_{y} \in{ }_{B} E_{y}^{\prime}$. Then we have

$$
l / h \geqq K^{-1}\left(l^{\prime 2} / F\right) .
$$

For $l=0, l^{\prime}>0$ we get $h=0$, i.e. the above theorem. For $l^{\prime}=\infty$ (we have to replace $l^{\prime}-\epsilon$ by a number $<l^{\prime}$ in the proof) and $l=a$ we get $h=0$.

COROLlary. The set of values $y$ for which the image curve of the segment $\Im z=y$ is not rectifiable is of measure zero. 
THEOREM 3. If $E$ is a closed subset of $G$ of two-dimensional measure zero and $w(z)$ any quasiconformal mapping of $G$, its maximal dilation on $G-E$ is equal to its maximal dilation on $G$. In other words, a closed. set of zero area is deletable with respect to all quasiconformal mappings of $G$.

Proof. We consider any analytic quadrilateral $Q \subset G$ and map it as well as its image $Q^{\prime}$ conformally onto the rectangles $R$ and $R^{\prime}$ respectively. As the set $E_{1}$ in $R$ which corresponds to the part $E \cap Q$ of the exceptional set $E$ in $G$ is closed and of zero area, the conditions of Lemma 3 are fulfilled. From Lemma 3 we conclude that the conditions of Lemma 2 with the constant $K_{0}$ are fulfilled. From Lemma 2 we get therefore

$$
a^{\prime} / b^{\prime} \leqq K_{0}(a / b)
$$

5. Another application of the same method leads to the following Lemma $3^{\prime \prime}$ : A topological mapping of $R$ onto $R^{\prime}$ with the property (1) of Lemma 3 is absolutely continuous on almost every horizontal line.

The set of all $y$ for which the length $L_{\eta}(\xi)$ of the image of the stretch $(0=x=\xi, y=\eta)$ is not absolutely continuous in $\xi$ is measurable. If it were not of measure zero, there would exist a closed set $B$ on the interval $0 \leqq y \leqq b$ of positive linear measure $h$ and a number $l$ such that for every $y$ in $B$ the corresponding horizontal stretch carries a system of intervals of total length $<\epsilon$ while their images have total length $\geqq l$. The rest of the proof is a repetition of the one given for Lemma 3 . Lemma $3^{\prime \prime}$ proves the theorem on absolute continuity of quasiconformal mappings which was announced in the Bull. Amer. Math. Soc. Abstract 61-3-421, by the same author.

\section{REFERENCES}

1. L. Ahlfors, On quasiconformal mappings, Journal d'Analyse Mathématique vol. $3(1953 / 1954)$ pp. 1-58.

2. L. Ahlfors and A. Beurling, Conformal invariants and function-theoretic null-sets, Acta Math. vol. 83 (1950) pp. 101-129.

Institute for Advanced Study 